\title{
MOMENT CAPACITY OF STEEL FIBER REINFORCED CONCRETE BEAMS
}

\author{
Muhammad I. M. Rjoub, Ph. D. \\ Department of Civil Engineering, Faculty of Engineering Technology, \\ Marka P.O. Box 15008, Amman 11134 Jordan \\ e-mail:m_rjoub1@hotmail.com
}

(Received October 17, 2005 Accepted December 14, 2005)

\begin{abstract}
Using discrete steel fibers in reinforced concrete improves some mechanical properties of concrete such as the tensile strength, shear strength, toughness and the flexural strength. In this research work, the effect of adding steel fibers on the moment capacity of steel fiber reinforced concrete (SFRC) is studied and new relationships accounting for the variations in the modulus of rupture resulted from the added steel fibers are presented. The relations are expressed in terms of the fiber content, fiber aspect ratio and the concrete compressive strength and then employed in predicting the moment capacity of SFRC beams.
\end{abstract}

The applicability of the proposed formula in predicting the moment capacity of SFRC beams is checked by analyzing fifty-two beams of different fiber content, concrete strengths and densities ranging from normal to light-weight. The comparisons of the predictions agreed with the corresponding experimental values.

KEYWORDS: Concrete; Flexural Strength; Steel fibers; Reinforced Concrete; SFRC

\section{INTRODUCTION}

Steel fiber reinforced concrete (SFRC) is primarily made of concrete and discrete steel fibers. The role of the randomly oriented, discontinuous fibers is to bridge across the cracks that develop in concrete. So, existence of steel fibers in concrete members improves the tensile strength, ductility and toughness in addition to the flexural strength. It is also recognized that replacing shear reinforcement by steel fibers enhances the shear strength of such members. Since the fibers are randomly distributed, the improvements in flexural strength resulting from the steel fibers are not large enough to fully substitute the continuous bars in flexural reinforced elements. So, a lot of researches have been carried out, over the past thirty years, on combining the SFRC and steel bars to achieve optimum conditions in flexural elements through using steel fibers together with the longitudinal steel bars [1]. 


\section{RESEARCH SIGNIFICANCE}

This work studies the effect of fiber content on the modulus of rupture of SFRC members, which in role affects the flexural capacity of such composites. The current research is directed to obtain a new formula capable to predict the flexural capacities of SFRC beams reinforced with longitudinal steel bars.

\section{FLEXURAL CAPACITY OF SFRC BEAMS}

Several methods that predict the flexural strength of SFRC beams of no longitudinal steel bars are found in the literature [2-5]. Most of them basically use the direct tensile strength, the split strength or the compressive strength in estimating the flexural capacity of SFRC members. Some of such methods are listed in Table 1.

Table1: Summary of methods that predict the flexural strength of SFRC.

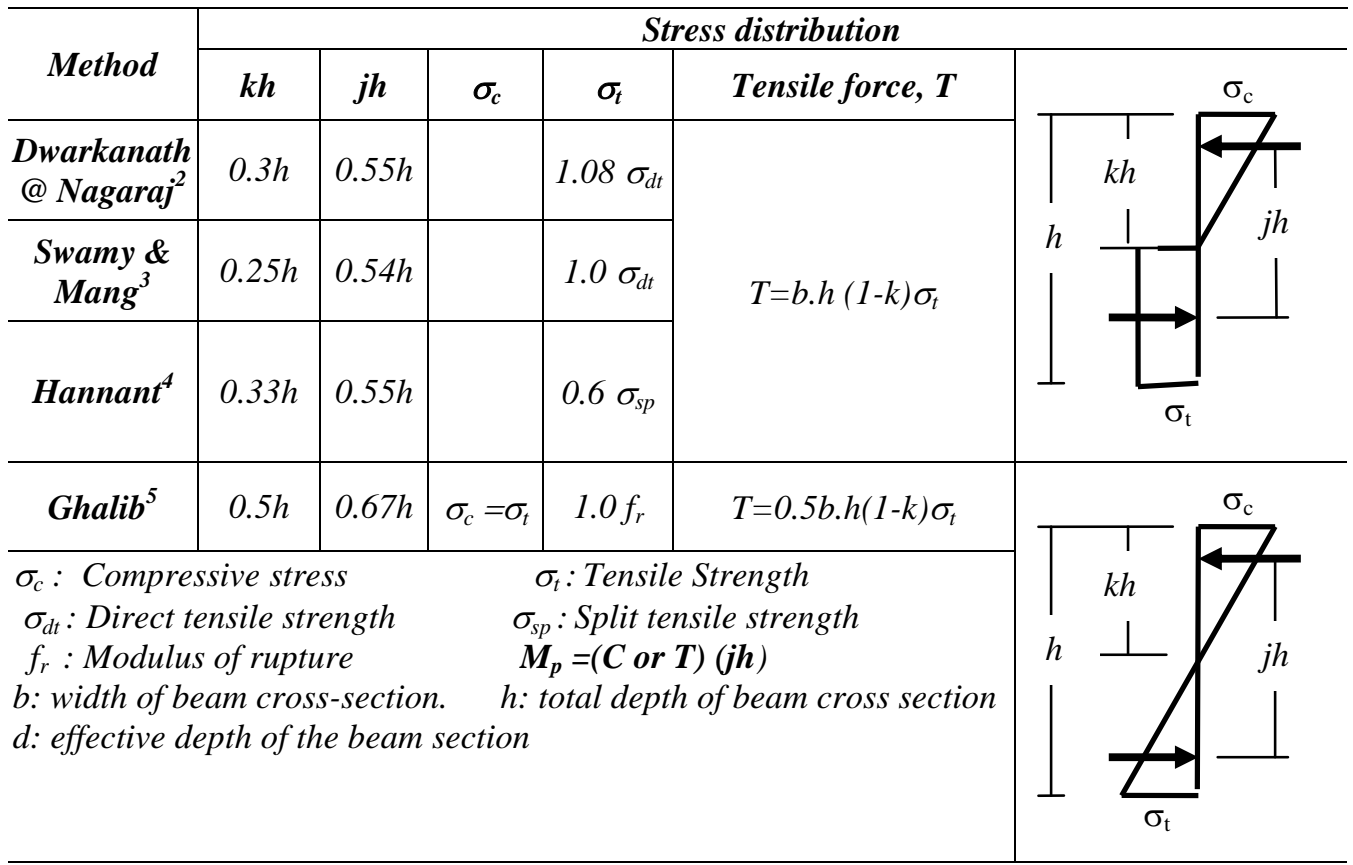

On the other hand, the design considerations for steel fiber concrete, ACI 544.4R [6] suggests the nominal moment capacity of SFRC section shown in Fig. 2 to be given by the following equations

$$
\begin{array}{lr}
M_{n}=A_{s} \cdot f_{y}\left(d-\frac{a}{2}\right)+0.5(h+e-a) \cdot b \cdot(h-e) \cdot \sigma_{t} & (1-a) \\
e=\left[0.003+\varepsilon_{t}(\text { fibers })\right] \frac{c}{0.003} & (1-b) \\
\sigma_{t}=0.00777 \mathfrak{z}_{f}(l / d) \cdot F_{b} & (1-c)
\end{array}
$$

Where: 
$\sigma_{t}$ is the tensile strength of the fibrous concrete.

$\mathrm{F}_{\mathrm{b}}$ is the bond efficiency of the fiber which varies from 1 to 1.2 depending on the fiber characteristics

$A_{s} \cdot f_{y}$ is tensile force in the steel reinforcement.

$v_{f}$ and $l / d$ are the fiber volumetric and aspect ratios, respectively

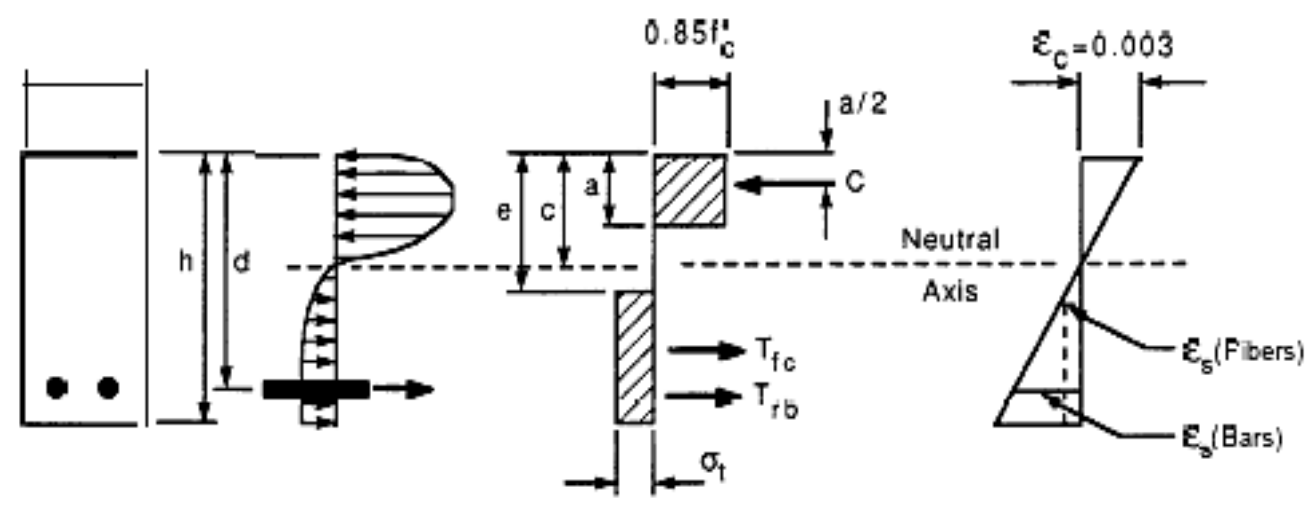

\section{Assumed Stress Distribution}

Simplified

Representation
Strain Diagram

Fig. 1: Design assumptions of reinforced concrete beams containing steel fibers [6].

The current research work, assumes the moment capacity of SFRC beams with steel bars to be composed of two parts: the first is the moment capacity of the conventional reinforced concrete $\left(M_{p}\right)$ and the second is the moment caused by the increase in the modulus of rupture of the SFRC beams resulted from the added steel fibers $\left(M_{f}\right)$. Referring to Fig. $2 b$ and $2 c$, the two parts can be expressed as follows:

$$
\begin{aligned}
& M_{f}=\Delta f_{r} \cdot b \cdot h(0.67 h) / 4=0.167 \Delta f_{r} \cdot b \cdot h^{2} \\
& M_{c}=A_{s} \cdot f_{y}\left(d-\frac{a}{2}\right) \\
& M_{p}=M_{c}+M_{f}
\end{aligned}
$$

Where

$$
a=A_{s} \cdot f_{y} /\left(0.85 f^{\prime}{ }_{c} \cdot b\right)
$$

$A_{s}$ and $f_{y}$ are the area and the yield strength of longitudinal steel bars, respectively. $\Delta f_{r}$ is the increase in the modulus of rupture caused by the presence of steel fibers. 

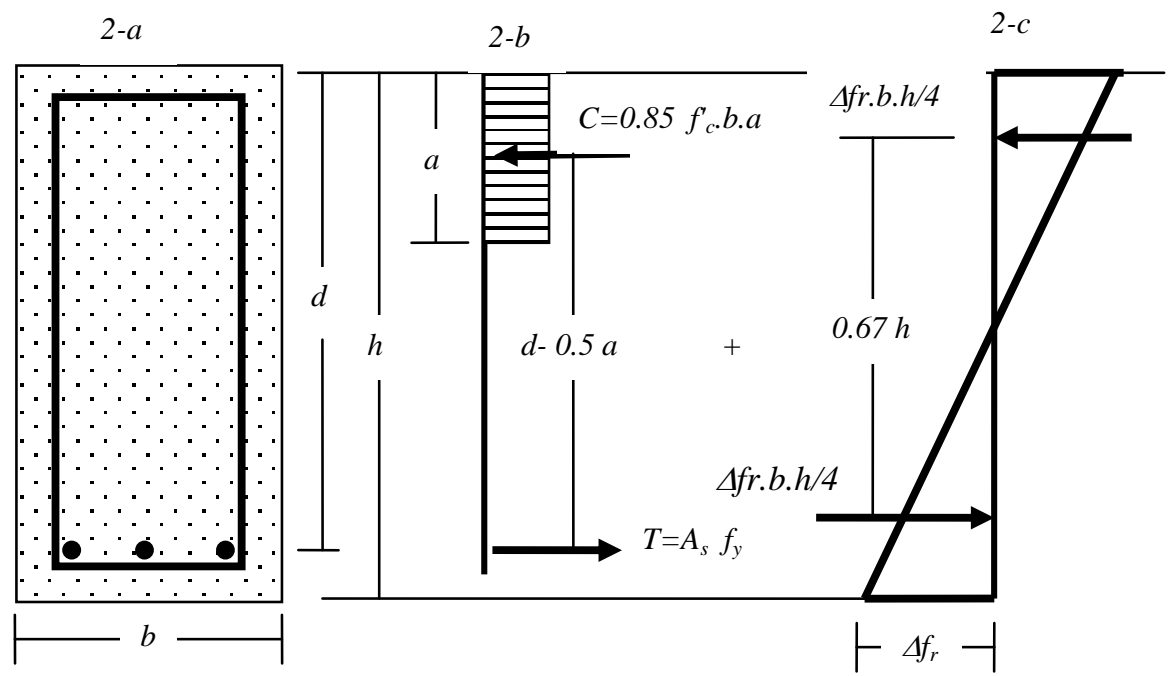

Fig. 2: Flexural analysis of SFRC beams with longitudinal steel.
(a) Beam cross-section
(b) Reinforced concrete contribution
(c) Fiber contribution.

In order to investigate the effect of the fiber reinforcement index on the modulus of rupture, 40 tests of the modulus of rupture, are gathered from literature [7-11] and analyzed using the regression analysis. The analyzed specimens, listed in Table 2 , have different concrete densities, concrete grades, fiber volumetric ratios and different fiber aspect ratios. The increase in the modulus of rupture, $\Delta f_{r}$ that represents the difference between the modulus of rupture of SFRC, $f_{r}$ and that of plain (no fiber) concrete, $f_{p}$ is reported in Table 2. The ratio $\Delta f_{r} / f_{p}$ is plotted against $v_{f}(l / d)$ as shown in Fig. 3 and the following relationships are obtained using the linear and nonlinear regression

$$
\begin{aligned}
& \Delta f_{r}=\left[0.61\left(v_{f} \cdot l / d\right)\right] f_{p} \\
& \text { (Linear regression) } \\
& \Delta f_{r}=\left[0.21\left(v_{f} \cdot l / d\right)^{2}+0.36\left(v_{f} l / d\right)\right] f_{p} \text { (Nonlinear regression) }
\end{aligned}
$$

Where the modulus of rupture, $f_{p}$ of the plain concrete is estimated by the ACI $318 \mathrm{M}$ 05 Building Code [17] such that $f_{p}$ equals $0.7 \lambda \sqrt{f_{c}^{\prime}}$ where the parameter $\lambda$ equals 1 for normal-weight concrete (NWC), 0.85 for sand light-weight concrete (LWC) and 0.75 for all LWC.

The applicability of the new method in predicting the flexural capacity of SFRC sections is checked by analyzing 52 beams tested experimentally by different researchers [12-15]. The analysis is conducted using Eqn 2 in connection with Eqn.3. Table 3 lists the basic information of the analyzed beams. The beams have different fiber content, different densities (normal weight and light-weight concrete) and their compressive strengths range from 16 to $90.5 \mathrm{MPa}$. The predicted moment capacities, $M_{p}$ of the analyzed beams are compared with the corresponding experimental results, 
$M_{\text {exp }}$ and plotted on Fig. 4. The plot of the predicted versus the experimental moment capacity shows acceptable predictions for the moment capacities using the proposed approach for both the NWC as well as the LWC beams.

Table 2: Modulus of rupture of SFRC composites.

\begin{tabular}{|c|c|c|c|c|c|c|}
\hline $\begin{array}{c}1 \\
\text { Author }\end{array}$ & $\begin{array}{c}2 \\
v_{f} \% \\
\end{array}$ & $\begin{array}{c}3 \\
l / d\end{array}$ & $\begin{array}{c}4 \\
F_{c}{ }_{c}[M P a]\end{array}$ & $\begin{array}{c}5 \\
f_{p}[M P a]\end{array}$ & $\begin{array}{c}6 \\
f_{r}[M P a]\end{array}$ & $\begin{array}{c}7 \\
\Delta f_{r} / f_{p}\end{array}$ \\
\hline \multirow{4}{*}{$\begin{array}{c}\text { Dwarkanath }^{2} \\
\text { (1991) }\end{array}$} & 0 & & - & 4.36 & - & 0 \\
\hline & 1 & 72 & - & & 6.64 & 0.522 \\
\hline & 2 & 72 & - & & 9.06 & 1.08 \\
\hline & 3 & 72 & - & & 11.74 & 1.69 \\
\hline \multirow{5}{*}{$\begin{array}{c}\text { Abdel- } \\
\text { Wahab }^{7} \\
(1997) \\
\text { (NSC) }\end{array}$} & 0 & & 19.57 & 2.9 & - & 0 \\
\hline & 0.5 & 62.5 & 22.63 & & 3.4 & 0.17 \\
\hline & 1 & 62.5 & 22 & & 3.95 & 0.36 \\
\hline & 1 & 62.5 & 19.84 & & 3.63 & 0.25 \\
\hline & 1 & 62.5 & 28.48 & & 3.25 & 0.12 \\
\hline \multirow{4}{*}{$\begin{array}{c}\text { Ashour and } \\
\text { Wafa }^{13} \\
(1993) \\
(H S C)\end{array}$} & 0 & & 86.14 & 8.93 & & 0 \\
\hline & 0.5 & 75 & 87.11 & & 9.94 & 0.11 \\
\hline & 1 & 75 & 88.11 & & 10.6 & 0.19 \\
\hline & 1.5 & 75 & 90.5 & & 13.64 & 0.53 \\
\hline \multirow{3}{*}{$\begin{array}{c}\text { Ashour and } \\
\text { Wafa }^{14} \\
(1997)\end{array}$} & 0 & 75 & 80.19 & 8.97 & & 0.0 \\
\hline & 0.75 & 75 & 80.87 & & 9.84 & 0.10 \\
\hline & 1.5 & 75 & 82.32 & & 14.05 & 0.57 \\
\hline \multirow{4}{*}{$\begin{array}{c}\text { Campione } \\
\text { and Papia }^{8} \\
(2001) \\
(L W C)\end{array}$} & 0 & & 20.6 & 4.1 & & 0 \\
\hline & 0.5 & 60 & 20.6 & & 4.3 & 0.05 \\
\hline & 1 & 60 & 20.6 & & 4.7 & 0.15 \\
\hline & 2 & 60 & 20.6 & & 5.85 & 0.43 \\
\hline \multirow{4}{*}{$\begin{array}{c}\text { Rjoub \& } \\
\text { Hunaiti }^{9} \\
(2002) \\
(N W C) \\
\end{array}$} & 0 & - & 25.1 & 3.73 & - & 0 \\
\hline & 0.5 & 75 & 25.0 & & 4.53 & 0.21 \\
\hline & 1 & 75 & 27.1 & & 4.8 & 0.29 \\
\hline & 1.5 & 75 & 29.6 & 4.1 & 8 & 0.95 \\
\hline \multirow{8}{*}{$\begin{array}{c}\text { Bani Yasin }{ }^{10} \\
(2004) \\
(\text { cubes } \\
100 \mathrm{~mm})\end{array}$} & 0 & & 23.74 & 6.96 & & 0 \\
\hline & 0.5 & 60 & 25.13 & & 7.88 & 0.13 \\
\hline & 1 & 60 & 26.6 & & 8.45 & 0.21 \\
\hline & 1 & 75 & 26.17 & & 9.25 & 0.33 \\
\hline & 0 & & 51.72 & 9.26 & & 0 \\
\hline & 0.5 & 60 & 54.83 & & 11.2 & 0.21 \\
\hline & 1 & 60 & 56.12 & & 14.8 & 0.60 \\
\hline & 1 & 75 & 55.58 & & 16.06 & 0.73 \\
\hline \multirow{8}{*}{$\begin{array}{c}\text { Aqayleh }^{11} \\
(2004) \\
\text { (cubes } \\
\text { 100mm) }\end{array}$} & 0 & 60 & 21.99 & 6.33 & & 0.00 \\
\hline & 0.5 & 60 & 22.43 & & 7.03 & 0.11 \\
\hline & 1 & 60 & 23.48 & & 7.32 & 0.16 \\
\hline & 1.5 & 60 & 24.94 & & 8.17 & 0.29 \\
\hline & 0 & 60 & 52.85 & 9.13 & & 0.00 \\
\hline & 0.5 & 60 & 54.16 & & 10.51 & 0.15 \\
\hline & 1 & 60 & 55.22 & & 11.43 & 0.25 \\
\hline & 1.5 & 60 & 56.46 & & 14.8 & 0.62 \\
\hline
\end{tabular}




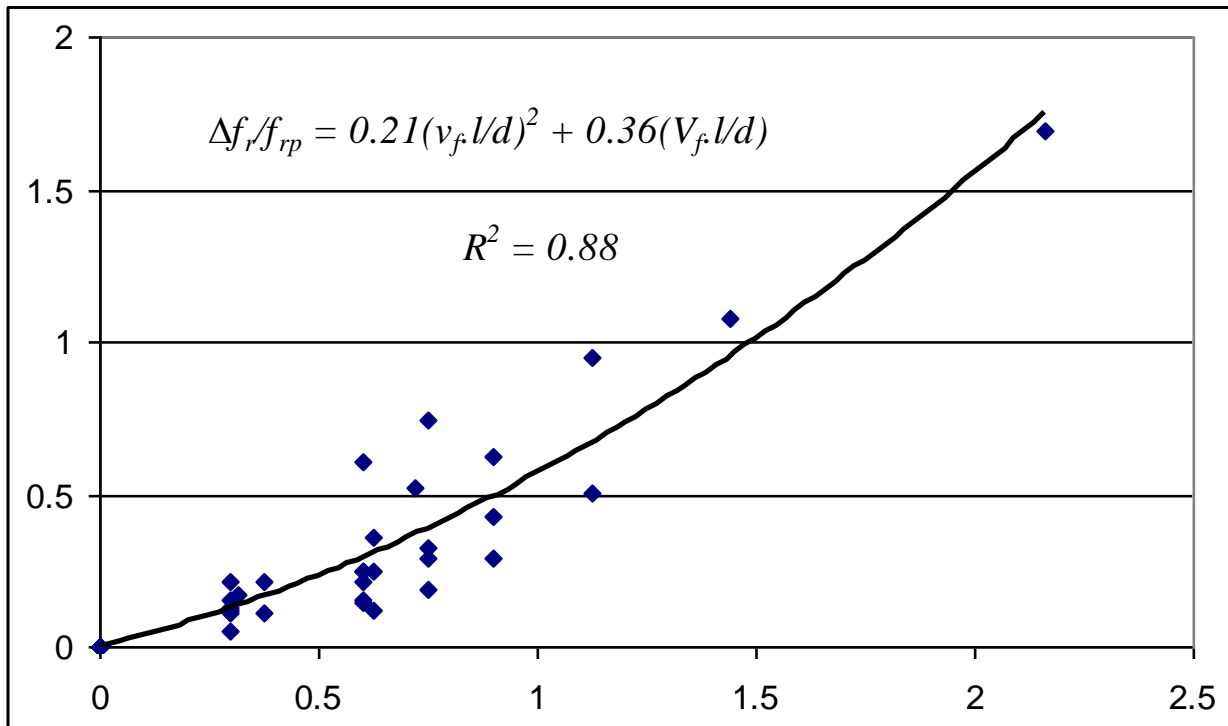

a. Effect of fiber reinforcement index on the modulus of rupture of SFRC [Nonlinear regression].

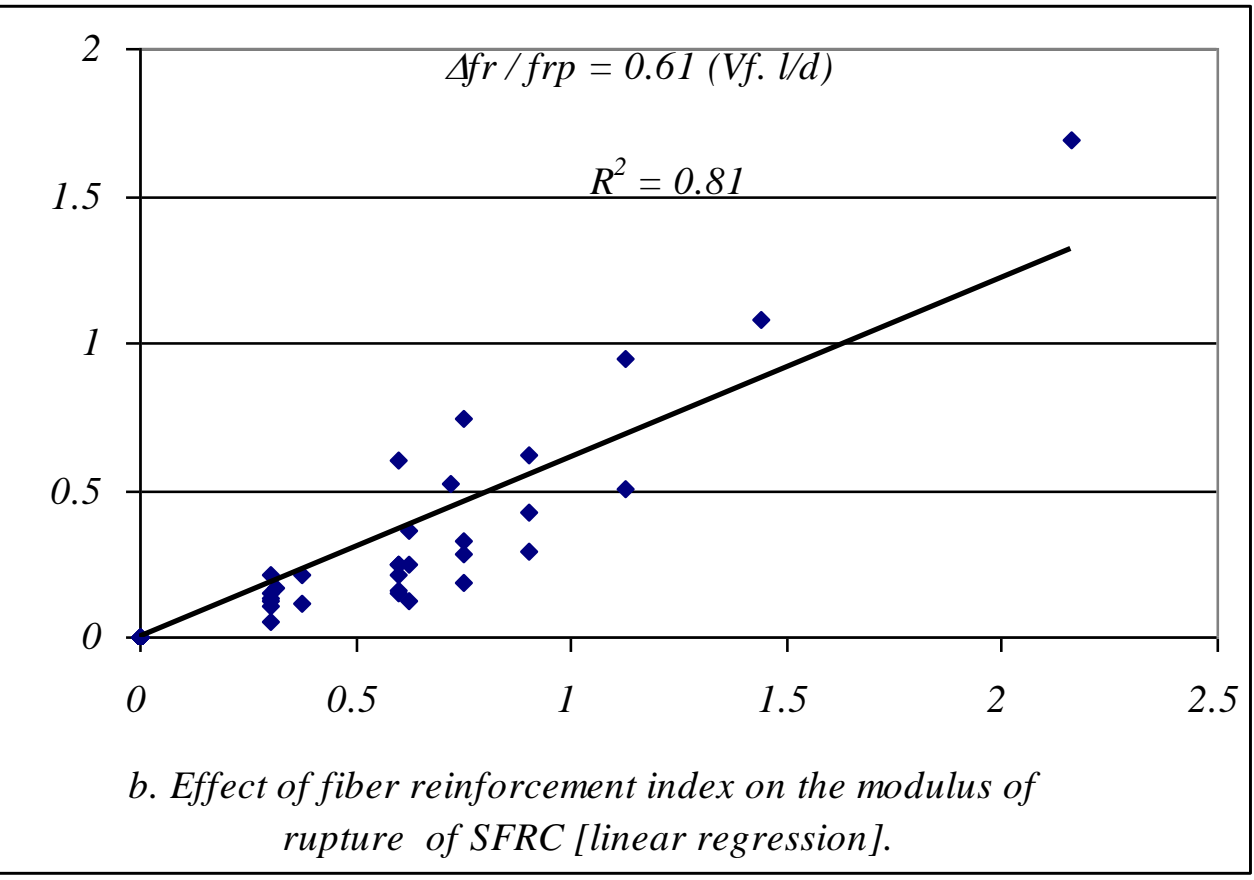

Fig. 3: Effect of fiber reinforcement index on the splitting strength of SFRC:
a. Nonlinear regression.
b. Linear regression 
Table 3: Flexural strength of SFRC beams (with longitudinal bars)

\begin{tabular}{|c|c|c|c|c|c|c|c|c|c|c|c|}
\hline \multirow{2}{*}{$\begin{array}{c}\text { Author } \\
\text { (Concrete } \\
\text { Type) }\end{array}$} & \multirow[t]{2}{*}{ Beam \# } & \multicolumn{3}{|c|}{$\begin{array}{c}\text { Cross section } \\
\text { dimensions }\end{array}$} & \multicolumn{5}{|c|}{ Material Properties } & \multirow{2}{*}{$\begin{array}{c}M_{\exp } \\
{[k N . m]}\end{array}$} & \multirow{2}{*}{$\begin{array}{c}M_{p} \\
{[k N . m]}\end{array}$} \\
\hline & & $\begin{array}{c}b \\
{[\mathrm{~mm}]}\end{array}$ & $\begin{array}{c}d \\
{[\mathrm{~mm}]}\end{array}$ & $\begin{array}{c}h \\
{[\mathrm{~mm}]}\end{array}$ & $\begin{array}{c}f^{\prime} c \\
{[M P a]}\end{array}$ & $\begin{array}{c}A_{s} \\
{\left[\mathrm{~mm}^{2}\right]}\end{array}$ & $\begin{array}{c}f_{y} \\
{[M P a]}\end{array}$ & $V_{f}$ & $l / d$ & & \\
\hline \multirow{18}{*}{$\begin{array}{c}\text { Abu } \\
\text { Hijab }^{12} \\
(\text { NWC) } \\
1999\end{array}$} & N1 & 200 & 208 & 250 & 30.46 & 308 & 415 & 0 & 75 & 27.34 & 25.01 \\
\hline & $\mathrm{N} 2$ & 200 & 208 & 250 & 30.46 & 308 & 415 & 0 & 75 & 26.75 & 25.01 \\
\hline & N3 & 200 & 208 & 250 & 30.38 & 308 & 415 & 0 & 75 & 28.65 & 25.00 \\
\hline & N4 & 200 & 208 & 250 & 30.38 & 308 & 415 & 0 & 75 & 24.26 & 25.00 \\
\hline & N5 & 200 & 258 & 300 & 30.63 & 308 & 415 & 0 & 75 & 36.00 & 31.41 \\
\hline & N6 & 200 & 258 & 300 & 30.63 & 308 & 415 & 0 & 75 & 35.82 & 31.41 \\
\hline & N7 & 200 & 208 & 250 & 30.46 & 308 & 415 & 0.5 & 75 & 28.99 & 27.14 \\
\hline & N8 & 200 & 208 & 250 & 30.46 & 308 & 415 & 0.5 & 75 & 29.43 & 27.14 \\
\hline & N9 & 200 & 208 & 250 & 30.38 & 308 & 415 & 0.5 & 75 & 29.44 & 27.13 \\
\hline & N10 & 200 & 208 & 250 & 30.38 & 308 & 415 & 0.5 & 75 & 26.23 & 27.13 \\
\hline & N11 & 200 & 258 & 300 & 30.63 & 308 & 415 & 0.5 & 75 & 37.20 & 34.48 \\
\hline & N12 & 200 & 258 & 300 & 30.63 & 308 & 415 & 0.5 & 75 & 36.72 & 34.48 \\
\hline & N13 & 200 & 208 & 250 & 30.46 & 308 & 415 & 1 & 75 & 30.44 & 29.27 \\
\hline & N14 & 200 & 208 & 250 & 30.46 & 308 & 415 & 1 & 75 & 30.28 & 29.27 \\
\hline & N15 & 200 & 208 & 250 & 30.38 & 308 & 415 & 1 & 75 & 30.77 & 29.26 \\
\hline & N16 & 200 & 208 & 250 & 30.38 & 308 & 415 & 1 & 75 & 28.85 & 29.26 \\
\hline & N17 & 200 & 258 & 300 & 30.63 & 308 & 415 & 1 & 75 & 37.68 & 37.56 \\
\hline & N18 & 200 & 258 & 300 & 30.63 & 308 & 415 & 1 & 75 & 37.80 & 37.56 \\
\hline \multirow{18}{*}{$\begin{array}{c}\text { Abu } \\
\text { Hijab }^{12} \\
(\mathrm{LWC} \\
\lambda=0.85) \\
1999\end{array}$} & & 200 & 208 & 250 & 10.04 & 308 & 415 & 0 & 75 & 22.50 & 21.80 \\
\hline & L20 & 200 & 208 & 250 & 10.04 & 308 & 415 & 0 & 75 & 22.70 & 21.80 \\
\hline & L21 & 200 & 208 & 250 & 10.04 & 308 & 415 & 0 & 75 & 25.20 & 21.80 \\
\hline & L22 & 200 & 208 & 250 & 10.04 & 308 & 415 & 0 & 75 & 25.00 & 21.80 \\
\hline & L23 & 200 & 158 & 200 & 10.04 & 308 & 415 & 0 & 75 & 16.10 & 15.41 \\
\hline & L24 & 200 & 158 & 200 & 10.04 & 308 & 415 & 0 & 75 & 16.00 & 15.41 \\
\hline & L25 & 200 & 208 & 250 & 13.70 & 308 & 415 & 0.5 & 75 & 27.00 & 24.29 \\
\hline & L26 & 200 & 208 & 250 & 13.70 & 308 & 415 & 0.5 & 75 & 27.50 & 24.29 \\
\hline & L27 & 200 & 208 & 250 & 13.70 & 308 & 415 & 0.5 & 75 & 28.80 & 24.29 \\
\hline & L28 & 200 & 208 & 250 & 13.70 & 308 & 415 & 0.5 & 75 & 29.00 & 24.29 \\
\hline & L29 & 200 & 158 & 200 & 13.70 & 308 & 415 & 0.5 & 75 & 18.20 & 17.46 \\
\hline & L30 & 200 & 158 & 200 & 13.70 & 308 & 415 & 0.5 & 75 & 18.80 & 17.46 \\
\hline & L31 & 200 & 208 & 250 & 15.44 & 308 & 415 & 1 & 75 & 28.80 & 26.05 \\
\hline & L32 & 200 & 208 & 250 & 15.44 & 308 & 415 & 1 & 75 & 29.00 & 26.05 \\
\hline & L33 & 200 & 208 & 250 & 15.44 & 308 & 415 & 1 & 75 & 30.00 & 26.05 \\
\hline & L34 & 200 & 208 & 250 & 15.44 & 308 & 415 & 1 & 75 & 31.10 & 26.05 \\
\hline & L35 & 200 & 158 & 200 & 15.44 & 308 & 415 & 1 & 75 & 20.00 & 18.73 \\
\hline & L36 & 200 & 158 & 200 & 15.44 & 308 & 415 & 1 & 75 & 19.60 & 18.73 \\
\hline \multirow{8}{*}{$\begin{array}{c}\text { Ashour \& } \\
\text { Wafa }^{14} \\
1993\end{array}$} & B1 & 170 & 265 & 300 & 86.14 & 628 & 437 & 0 & 75 & 105.65 & 69.70 \\
\hline & B2 & 170 & 265 & 300 & 87.11 & 628 & 437 & 0.5 & 75 & 116.46 & 74.14 \\
\hline & B3 & 170 & 265 & 300 & 88.11 & 628 & 437 & 1 & 75 & 122.81 & 78.63 \\
\hline & B4 & 170 & 265 & 300 & 90.53 & 628 & 437 & 1.5 & 75 & 130.38 & 83.33 \\
\hline & B5 & 170 & 265 & 300 & 86.14 & 628 & 437 & 0 & 75 & 104.61 & 69.70 \\
\hline & B6 & 170 & 265 & 300 & 87.11 & 628 & 437 & 0.5 & 75 & 115.77 & 74.14 \\
\hline & B7 & 170 & 265 & 300 & 88.11 & 628 & 437 & 1 & 75 & 118.52 & 78.63 \\
\hline & B8 & 170 & 265 & 300 & 90.50 & 628 & 437 & 1.5 & 75 & 120.82 & 83.33 \\
\hline
\end{tabular}




\begin{tabular}{|c|c|c|c|c|c|c|c|c|c|c|c|}
\hline \multirow{3}{*}{$\begin{array}{l}\text { Ashour } \\
\text { et al }^{15} \\
\text { (1997) }\end{array}$} & B1-0 & 100 & 125 & 150 & 80.19 & 157 & 412 & 0 & 75 & 8.73 & 7.78 \\
\hline & B $1-0.75$ & 100 & 125 & 150 & 80.87 & 157 & 412 & 0.75 & 75 & 9.60 & 8.72 \\
\hline & $\mathrm{B} 1-1.5$ & 100 & 125 & 150 & 82.32 & 157 & 412 & 1.5 & 75 & 10.95 & 9.68 \\
\hline \multirow{5}{*}{$\begin{array}{c}\text { Craig }^{16} \\
1987\end{array}$} & BN1 & 177.8 & 324 & 381 & 32.75 & 1019 & 448.2 & 1.75 & 100 & 169.17 & 148.15 \\
\hline & $\mathrm{BN} 2$ & 177.8 & 324 & 381 & 40.34 & 1019 & 317.2 & 1.75 & 100 & 146.12 & 119.74 \\
\hline & $\mathrm{BN} 3$ & 177.8 & 324 & 381 & 34.48 & 1019 & 317.2 & 1.75 & 100 & 130.07 & 116.50 \\
\hline & BHS4 & 203.2 & 254 & 304.8 & 54.47 & 1019 & 448.2 & 0 & 100 & 105.44 & 104.95 \\
\hline & BHS5 & 203.2 & 254 & 304.8 & 68.95 & 1019 & 448.2 & 1.75 & 100 & 120.58 & 129.86 \\
\hline \multicolumn{4}{|c|}{$\begin{array}{c}\text { NWC: } \text { Normal weight concrete } \\
H S C: \text { High strength concrete }\end{array}$} & \multicolumn{6}{|c|}{ LWC: Light weight concret } & & \\
\hline
\end{tabular}

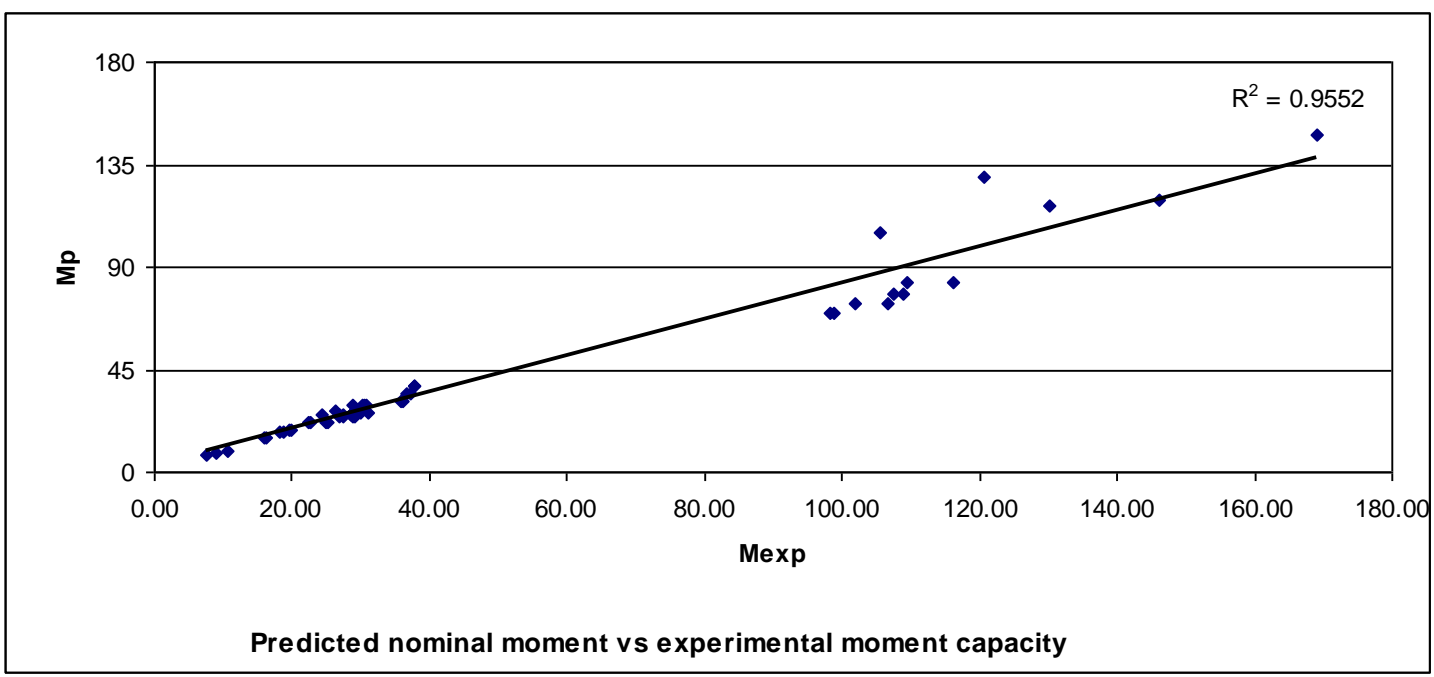

Fig. 4: Predicted versus experimental moment capacities of SFRC beams.

\section{CONCLUSIONS}

A method capable for predicting the moment capacity of SFRC beams with longitudinal tension steel bars is presented. The method adds the moment caused by the increase in the modulus of rupture of SFRC beams, in terms of the fiber reinforcement index to that adopted in the conventional RC beams. The comparisons of the experimental flexural moments with the predicted values showed acceptable predictions for both the normal and high strength concrete members whether they are constructed from NWC or LWC.

\section{REFERENCES}

[1] Hartmann, T. "Steel Fiber Reinforced Concrete," M. Sc. Thesis, Royal Institute of Technology in Stockholm, May 1999, $41 \mathrm{pp}$

[2] Dwarakanath, H. and Nagaraj, T., "Comparative Study of Predictions of Flexural Strength of Steel Fiber Reinforced Concrete," ACI-Structural J., Vol. 88 No. 6, Nov-Dec. 1991, pp. 714-720 
[3] Swamy, R., Mangant, P. and Rao, C., "Mechanics of Fiber Reinforcement of Cement Matrix," Fiber Reinforced Concrete, SP-44, American Concrete Institute, Detroit, 1973 pp 1-28

[4] Hannant D. "Fiber Cement and Fiber Concretes," John Wiley and Sons, New York, 1978, $219 \mathrm{pp}$.

[5] Ghalib, M. "Moment Capacity of Steel Fiber Reinforced Small Concrete Slabs," ACI-Structural J., Vol. 77 No. 4, July-Aug. 1980, pp. 247-257.

[6] ACI 544.4R88, "Design Considerations for Steel Fiber Reinforced Concrete," American Concrete Institute, Detroit 1999.

[7] Abdel Wahab, H. "Design of Fiber Reinforced Concrete Brackets," ACIStructural J., Vol. 94 No. 6, Nov-Dec. 1997, pp. 714-720

[8] Campione, G., Miraglia, N., and Papia, M., "Mechanical Properties of Steel Fiber Reinforced Lightweight Concrete with Pumice Stone or Expanded Clay Aggregates," Material and Structures, Vol. 34, May 2001, pp 201-210.

[9] Rjoub M. I., Huneiti Y., "Flexural Strength of Steel fiber Reinforced Concrete beams," The $6^{\text {th }}$ International Conference on Concrete Technology for Developing Countries, Amman-Jordan, October 2002, pp 519-530.

[10] Bani Yasin, I. S. "Performance of High Strength Fibrous Concrete Slab-column Connections Under Gravity and lateral loads," M. Sc. Thesis Jordan University for Science and Technology, Irbid, Jordan, June, 2004

[11] Aqayleh, "The Structural Behavior of High Strength Normal Weight Tow-Way Slabs With Steel Fibers," M. Sc. Thesis, Jordan University for Science and Technology, Irbid, Jordan, 2004

[12] Abu Hijab W. A. H.,"Flexural Strength of Lightweight and Normal Concrete Beams with Steel Fibers," M. Sc. Thesis, The University of Jordan, Jordan, August 1999, 112 pp.

[13] Ashour, S., and Wafa, F., "Flexural Behavior of High-Strength Steel Fiber Reinforced Concrete Beams," ACI-Structural J., Vol. 90 No. 3, May-June 1993, pp. 279-287

[14] Ashour S., Mahmoud K. and Wafa F., "Influence of Steel Fiber and Compression reinforcement on Deflection of High Strength Concrete Beams, ACI Structural J. Nov. Dec. 1997. pp 611-624

[15] Craig R. J. "Flexural Behavior and Design of Reinforced Concrete Members," Fiber Reinforced Concrete Properties and Applications, SP 105 American Concrete Institute, Detroit, 1987, 517-564.

[16] ACI 318-05, "Building Code Requirements for Reinforced Concrete and Commentary," American Concrete Institute, Farmington Hill, Mich. 2005. 


\title{
مقاومة الأنعطاف للجوائز الخرسانية المسلحة بالألياف
}

\author{
د. محمد ابراهيم الرجوب الري

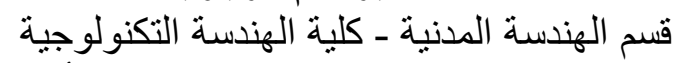 \\ ماركا- ص. ب. 15008- عمان 11134 الأردنة
}

e-mail: m_rjoub1@ @otmail.com

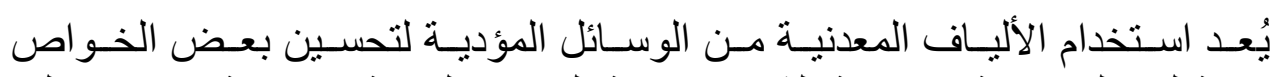

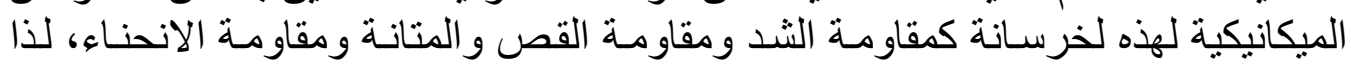

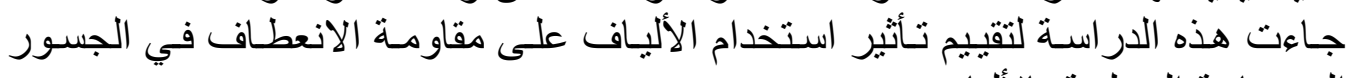
الخرسانية المسلحة بالألياف. لأفيم. وقد قدم هذا البحث علاقات جديدة أخذت بالحسبان التغير في معامل تمزف الخرسانة

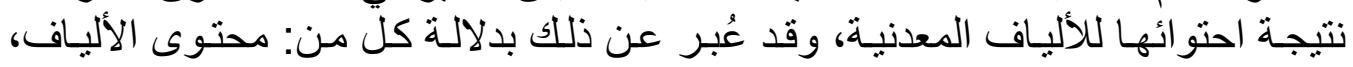

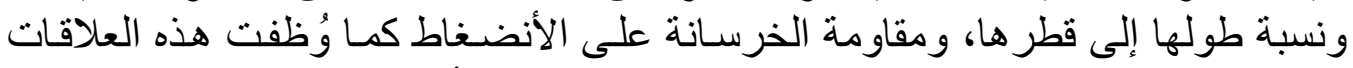

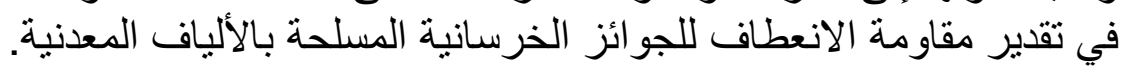

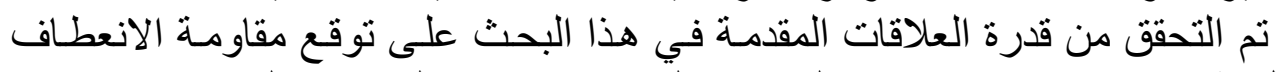

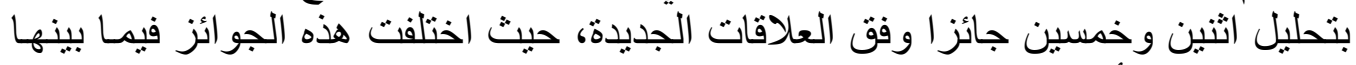

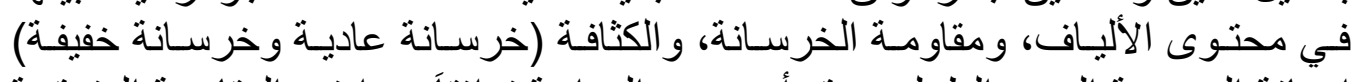

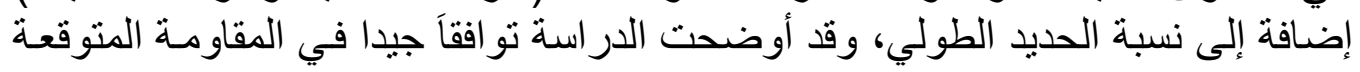
باستخدام العلاقات المقترحة مع القيم التجريبية المناظرة لهول لها. 